\title{
Unique role of admission hyperglycemia on myocardial infarction size and area at risk following an acute ST-elevation myocardial infarction
}

Taghreed Sayed Meshref ${ }^{1 *}$, Mahmoud Aly Ashry ${ }^{1}$, Refaat Fathi Abd El-Aal ${ }^{1}$, Hisham Mostafa Imam² and Dina Ali Hamad ${ }^{1}$

\begin{abstract}
Background: Hyperglycemia can adversely affect patients with acute ST-elevation myocardial infarction (STEMI) in both diabetic and non-diabetic patients. The majority of the studies had investigated the impact of admission hyperglycemia (AH) on cardiovascular morbidity and mortality while, in ours, we entailed its impact on final infarction size (FIS) and more interestingly, on the area at risk (AAR), both were estimated by cardiac magnetic resonance (CMR) imaging.
\end{abstract}

Results: AH showed significant positive correlations to FIS and AAR. Moreover, AH group had higher summation of ST segment elevation (sum STE), more maximum ST segment elevation (max STE), higher echocardiographic wall motion score index (WMSI), higher CMR estimated WMSI, and lower segmental ejection fraction (EF). Multivariate analysis showed that AH was independently associated with increased FIS.

Conclusion: Current study showed an association between AH and large FIS in STEMI patients.

Keywords: Admission hyperglycemia, ST-elevation myocardial infarction, Infarction size

\section{Background}

Accounting for more than 15 million deaths annually, cardiovascular diseases are the leading causes of death worldwide [1]. In the last decade, the mortality rates after ST-elevation myocardial infarction (STEMI) showed an obvious decline which is explained by the expanded use of primary percutaneous coronary intervention (PPCI), fibrinolytic therapies, and updated antithrombotics [1]. But, in Egypt, as well as low- and middle-income countries, mortality and cardiovascular events are still relatively high with the comparable burden with that of infectious diseases and other non-

\footnotetext{
*Correspondence: toti_20001000@hotmail.com

'Internal Medicine Department, Assiut University Hospitals, Faculty of

Medicine, Assiut University, Assiut 71511, Egypt

Full list of author information is available at the end of the article
}

communicable diseases, despite improvement in therapeutic and interventional therapy [1].

Admission hyperglycemia ( $\mathrm{AH})$ has been determined to inversely affect short- and long-term prognosis in patients with STEMI regardless of their diabetic state [2].

Single-photon emission computed tomography (SPEC $\mathrm{T}$ ) is the traditional imaging tool utilized to measure FIS, but it has a limited spatial resolution, and carry the risk of radiation [3]. CMR is a relatively new cardiac imaging modality that can assess changes in cardiac contractile function, metabolic activity, and membrane integrity. Furthermore, CMR has a high spatial and temporal resolution which facilitates accurate assessment of cardiovascular structures. By the addition of late gadolinium enhancement (LGE) with CMR, transmural extension and scar tissues can be estimated [4]. 
A few data about the sequelae of AH on FIS after acute STEMI patients are present and nearly no available data about the relation between $\mathrm{AH}$ and area at risk, and the objective of the current study was to investigate the relationship between admission blood glucose and myocardial FIS and AAR after reperfusion in STEMI patients by using CMR.

\section{Methods}

\section{Study population}

Forty-three patients who were presented with their first acute STEMI and underwent successful reperfusion, in a period less than $12 \mathrm{~h}$ from onset of symptoms, were included. They were selected from the Critical Care Unit, Internal Medicine Department, from April 2018 to May 2019. Patients were informed about the research and signed informed consent. Acute STEMI is diagnosed when there is chest pain lasting $\geq 30 \mathrm{~min}$; ST-segment elevation defined according to the European Society of Cardiology/ACCF/AHA as a new ST elevation at the J point affecting at least 2 contiguous leads of $\geq 2 \mathrm{~mm}$ in males or $\geq 1.5 \mathrm{~mm}$ in females in leads $\mathrm{V} 2-\mathrm{V} 3$ and/or of $\geq 1 \mathrm{~mm}$ in remaining contiguous chest or limb leads [5]. Successful reperfusion determined by resolution of STsegment $\geq 50 \% 1 \mathrm{~h}$ after thrombolysis, which can be classified into partial resolution [50-70\%] or complete resolution $[>70 \%]$.

Patients $\geq 85$ years old, with life-limiting non-cardiac disease, prior STEMI, left bundle branch block (LBBB), any contraindications to MRI, and an estimated glomerular filtration rate $<30 \mathrm{ml} / \mathrm{min} / 1.73 \mathrm{~m}^{2}$, who were hemodynamically unstable to be transferred to CMR laboratory were excluded.

According to $\mathrm{AH}$ they were divided into 2 groups (patients with $\mathrm{AH}=21$ and patients without $\mathrm{AH}=22$ ). $\mathrm{AH}$ was defined as the presence of random plasma glucose > $140 \mathrm{mg} / \mathrm{dl}$ in non-diabetic patients within $2 \mathrm{~h}$ of admission [5]. Plasma glucose levels (PG) were assessed every $2 \mathrm{~h}$; mean PG levels were calculated at days 1, 2, and 3 and just before CMR.

\section{Data Collection}

Demographic data including age, gender, body mass index, history of hypertension, diabetes mellitus, dyslipidemia, smoking, duration of chest pain, Killip class, and electrocardiogram (ECG) data were collected. Venous blood samples were taken on admission to measure glucose level and glycated hemoglobin (HbA1c). Low density lipoprotein (LDL) was assessed from fasting venous blood samples taken on the first morning after admission. Peak plasma values of creatine kinase $(\mathrm{CK})$ and $\mathrm{CK}-\mathrm{MB}$ were also evaluated. Baseline serum creatinine clearance was also evaluated by the Cockcroft-Gault formula [6].

\section{CMR protocol}

Cardiac MRI was performed 4 to 6 days after acute STEMI with a 1.5-Tesla scanner (Philips, Achieva) using a 6-channel body array coil. Left ventricular (LV) longaxis scout images were taken to adjust the LV short-axis imaging planes [7]. Short-axis slices (thickness of $5 \mathrm{~mm}$ with a slice gap of $10 \mathrm{~mm}$ ) were defined from the base to the apex of the heart. Imaging of the heart was performed at the end-systolic time to estimate left ventricular end-systolic volume (LVESV) and at the end-diastolic time to estimate left ventricular end-diastolic volume (LVEDV). Steady-state free precession sequences were used for cine imaging; a dark-blood T2-weighted shorttau inversion-recovery turbo-spin echo sequence (STIR) was applied to determine AAR (with edema), and a segmented phase-sensitive inversion-recovery (PSIR) steady-state free precession sequence was used for late enhancement imaging $10 \mathrm{~min}$ after intravenous injection of $0.15 \mathrm{mmol} / \mathrm{kg}$ of gadolinium diethylenetriaminepentaacetic acid (Gd-DTPA) (Magnevist; Bayer Schering, Berlin, Germany). All images were ECG-triggered and acquired during breath-holds. CMR studies were analyzed then offline (Philips Medical Systems, Nederland B.V).

\section{CMR data analysis}

Data were analyzed by using a 17-segment model. From short-axis views, both LVEDV and LVESV were measured. Left ventricular ejection fraction (LVEF) was calculated as LVEF $=($ EDV - ESV $) / E D V$.

For each patient, AAR was recognized as hyper-intense zones on T2-weighted images; left ventricular endo- and epicardial borders had been delineated; then, reference normal area was drawn. AAR was defined as the percentage of LV volume delineated by the hyper-intense zone on T2-STIR images with signal intensity was $>2$ standard deviations above the mean signal obtained in the distant non-infarcted myocardium and was expressed as a percentage of normal myocardium. FIS was measured on PSIR sequences by delineating epicardial and endocardial borders; the papillary muscle was considered as a part of the cavity of the left ventricle; then, a myocardial area was regarded as hyper-intense whenever the signal intensity was $>2$ standard deviations above the mean signal intensity in the non-infarcted myocardium; then, the FIS was expressed as a percentage of normal non-infarcted myocardium (Fig. 1).

\section{Statistical analysis}

IBM-SPSS 21 was used for the analysis of data. The normality was tested by the Kolmogorov-Smirnov test. The continuous variables were presented as the means \pm standard deviation (SD), and categorical variables were expressed in the form of percentages. The difference in 

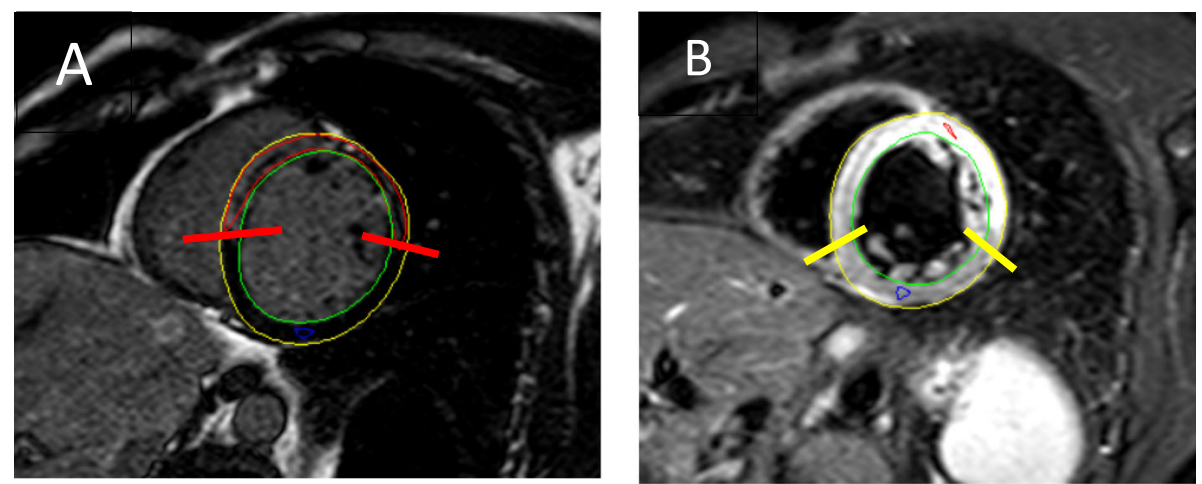

Fig. 1 Cardiac MRI at 1 week in a patient with a large anterior myocardial infarction. a Late enhancement imaging allowed for the definition of infarct size (hyper-enhanced myocardium, between red arrows). B T2-weighted sequence demonstrated a large area at risk (with edema, between yellow arrows)

frequencies among different groups was compared by Chi-square which was used to test differences in categorical data between groups, while Student $t$ test and MannWhitney $U$ test were used to test differences in means in continuous variables between groups (parametric and non-parametric). Spearman rank correlation coefficient was calculated for univariate correlations between echo and CMR parameters. A $p$ value of less than 0.05 was considered significant. Multivariate linear regression analysis was performed to test the independent predictors of FIS.

\section{Results}

\section{Clinical and echocardiographic characteristics}

The data showed that male is the predominate gender (86.4\% of the euglycemic group and $81 \%$ of AH group) with insignificant differences between both groups as regards mean age ( $p$ value $=0.487)$ and gender $(p$ value $=$ 0.473). Patients with AH had a significantly longer chest pain duration, higher heart rate on discharge, higher percent of anterior MI, higher summation of ST elevations (sum STE) on admission and maximum ST-segment elevations ( $\max$ STE) on admission, and higher baseline echocardiography-derived classic WMSI (Table 1).

In comparing PG levels between both groups, there was insignificant difference in all days before CMR performance; only they were significantly different in admission plasma glucose levels (Table 2).

\section{CMR findings}

Although there were no differences in LVEDV, LVESV, and EF between the two groups, the AH group had significantly higher classic WMSI, larger FIS, and AAR (Table 3).

\section{Correlation analysis}

Figure 2 shows that there was a significant positive correlation between admission plasma glucose (APG), FIS, and AAR in patients with STEMI.

\section{Regression analysis}

The multivariate linear regression analysis of the significant factors affecting myocardial CMR LGE infarction size. After adjusting for age, the final linear regression model contained six predictors: chest pain duration, APG, peak CK-MB, sum ST, max ST, and WMSI estimated by CMR (Table 4).

\section{Discussion}

Despite improvements in medical and interventional therapies in the last 40 years, acute STEMI is still a leading cause of mortality and morbidity. Many studies were done to determine possible factors that may increase poor outcomes in STEMI patients, mostly following myocardial remodeling. Admission hyperglycemia is a unique factor deserving a great interest to investigate its role in the outcomes of STEMI patients, with special emphasis on its relation to FIS and AAR.

Roles of hyperglycemia in patients with STEMI have many points of interest, first, chronic hyperglycemia which present even before AMI in diabetic patients, increased myocardial susceptibility to infarction in DM patients was indicated by two clinical studies, which detected significantly larger infarct size measured by SPECT in diabetic than non-diabetic patients [8]. This can be explained by impaired collateral recruitment, microcirculatory abnormalities, and underuse of evidence-based therapies [9]; second, hyperglycemia during hospital stay, and lastly and more interestingly, $\mathrm{AH}$ which is a unique predictor studied by our research and attracted more attention to the need of more strict control of admission plasma glucose.

Egypt has nearly 100 million people who have special demographic and social characteristics which increase the possibility of the stress-induced conditions and its health-associated problems; some of these characteristics 
Table 1 Patients' clinical and echocardiographic characteristics

\begin{tabular}{|c|c|c|c|}
\hline & Euglycemic, $n=22$ & Admission hyperglycemia, $n=21$ & $p$ value \\
\hline Age/years (mean \pm SD) & $56.49 \pm 11.4$ & $58.95 \pm 11.3$ & $0.487^{*}$ \\
\hline Sex (males\%) & 19 (86.4\%) & $17(81 \%)$ & $0.473^{* *}$ \\
\hline Hypertension $n(\%)$ & $12(54.5 \%)$ & $9(42.9 \%)$ & $0.443^{* *}$ \\
\hline Smoking $n(\%)$ & $15(68.2 \%)$ & $11(52.4 \%)$ & $0.289^{* *}$ \\
\hline Chest pain duration/h (mean \pm SD) & $6.36 \pm 3.2$ & $9.81 \pm 5.2$ & $0.019 * * *$ \\
\hline BMI (kg/m2) & $26.64 \pm 4.8$ & $25.52 \pm 4.7$ & $0.448^{*}$ \\
\hline Heart rate (beat/min) & $84.09 \pm 14.0$ & $92.05 \pm 11.2$ & $0.050^{*}$ \\
\hline $\mathrm{SBP}(\mathrm{mm} / \mathrm{Hg})$ & $118.46 \pm 21.7$ & $122.38 \pm 23.2$ & $0.587^{*}$ \\
\hline $\mathrm{DBP}(\mathrm{mm} / \mathrm{Hg})$ & $75.91 \pm 11.2$ & $77.14 \pm 15.1$ & $0.767^{*}$ \\
\hline Killip score & & & $0.951^{*}$ \\
\hline Class 1 & 19 (86.4\%) & $18(85.7 \%)$ & \\
\hline Class II & $3(13.6 \%)$ & $3(14.3 \%)$ & \\
\hline Anterior $n(\%)$ & $7(31.8 \%)$ & $13(61.9 \%)$ & $0.048^{* *}$ \\
\hline Admission sum of STE/mm (mean \pm SD) & $11.09 \pm 9.8$ & $18.14 \pm 7.9$ & $0.002^{* * *}$ \\
\hline Admission max STE/mm (mean \pm SD) & $3.59 \pm 2.3$ & $5.10 \pm 1.8$ & $0.003^{* * *}$ \\
\hline Resolution of STE $n(\%)$ & & & $0.625^{* *}$ \\
\hline$\geq 50-70$ & $10(45.5 \%)$ & $8(38.1 \%)$ & \\
\hline$\geq 70$ & $12(54.5 \%)$ & $13(61.9 \%)$ & \\
\hline LDL (mg/dl) (mean \pm SD) & $108.41 \pm 35.5$ & $106.38 \pm 28.6$ & $0.874^{* * *}$ \\
\hline S. creatinine $(\mathrm{mg} / \mathrm{dl})($ mean $\pm \mathrm{SD})$ & $0.95 \pm 0.2$ & $1.01 \pm 0.2$ & $0.259^{* * *}$ \\
\hline Initial CK-MB (ng/ml) (mean \pm SD) & $57.14 \pm 36.9$ & $64.76 \pm 42.5$ & $0.381^{* * *}$ \\
\hline Peak CK-MB (ng/ml) (mean \pm SD) & $245.23 \pm 184.3$ & $306.38 \pm 174.2$ & $0.162^{* * *}$ \\
\hline HBA1C & $5.11 \pm 0.9$ & $5.50 \pm 0.9$ & $0.087^{* * *}$ \\
\hline Classic WMSI (mean \pm SD) & $1.38 \pm 0.3$ & $1.52 \pm 0.2$ & $0.042^{* * *}$ \\
\hline LVESV (ml) (mean \pm SD) & $41.71 \pm 13.7$ & $40.96 \pm 12.7$ & $0.356^{* * *}$ \\
\hline LVEDV $(m l)($ mean \pm SD) & $81.95 \pm 18.5$ & $75.60 \pm 15.9$ & $0.789 * * *$ \\
\hline $\mathrm{EF} \%($ mean $\pm \mathrm{SD})$ & $54.05 \pm 11.5$ & $50.81 \pm 12.0$ & $0.462^{* * *}$ \\
\hline
\end{tabular}

$p$ value $\leq 0.05$ is significant

$B M I$ body mass index, $C K$ creatinine kinase, DBP diastolic blood pressure, EF ejection fraction, HBA1c glycated hemoglobin, $L D L$ low density lipoprotein, $L V E D V$ left ventricular end diastolic volume, LVESV left ventricular end systolic volume, Max STE maximum ST-segment elevation, SBP systolic blood pressure, SD standard deviation, SUm of STE summation of ST-segment elevations, WMSI wall motion score index

*Independent $t$ test was used to compare the mean difference between groups

${ }^{* *}$ Chi-square test was used to compare the proportion differences

***Mann-Whitney $U$ test was used to compare the median difference between groups

Table 2 Comparison between the stress hyperglycemia group and the euglycemic group as regards plasma glucose levels in all days before cardiac magnetic resonance

\begin{tabular}{llll}
\hline & Euglycemic group (no. 22) & Stress hyperglycemic group (no. 21) & $p$ value \\
\hline Mean APG & 223.45 & 11.33 & $<0.0001$ \\
Day 1 mean PG level $(\mathrm{mg} / \mathrm{dl})$ & 142.64 & 121.44 & 0.09 \\
Day 2 mean PG level $(\mathrm{mg} / \mathrm{dl})$ & 122.64 & 11.38 & 0.06 \\
Day 3 mean PG level $(\mathrm{mg} / \mathrm{dl})$ & 115.36 & 114.28 & 0.17 \\
Mean PG level before just CMR $(\mathrm{mg} / \mathrm{dl})$ & 113.14 & 116.23 & 0.10 \\
\hline
\end{tabular}

$p$ value $<0.05$ is significant

$A P G$ admission plasma glucose, $C M R$ cardiac magnetic resonance, $P G$ plasma glucose

*Independent $t$ test was used to compare the mean difference between groups 
Table 3 Cardiovascular magnetic resonance imaging findings

\begin{tabular}{llll}
\hline & Euglycemic, $n=22$ & Admission hyperglycemia, $n=21$ & $p$ value* \\
\hline LVESV $(\mathrm{ml})$ & $75.42 \pm 21.2$ & $81.39 \pm 26.9$ & 0.360 \\
LVEDV $(\mathrm{ml})$ & $136.71 \pm 22.2$ & $140.89 \pm 24.1$ & 0.679 \\
EF \% & $51.09 \pm 12.8$ & $49.45 \pm 13.1$ & 0.590 \\
Classic WMSI & $1.39 \pm 0.3$ & $1.56 \pm 0.2$ & $\mathbf{0 . 0 2 1}$ \\
FIS \% & $19.73 \pm 9.5$ & $28.05 \pm 13.1$ & $\mathbf{0 . 0 2 2}$ \\
AAR \% & $31.59 \pm 13.4$ & $39.62 \pm 16.6$ & 0.135 \\
\hline
\end{tabular}

$p$ value $<0.05$ is significant. Data were expressed in mean $\pm \mathrm{SD}$

$A A R$ area at risk, EF ejection fraction, FIS final infarct size, LVEDV left ventricular end diastolic volume, LVESV left ventricular systolic volume, MVO microvascular obstruction, $S D$ standard deviation, WMSI wall motion score index

*Mann-Whitney $U$ test was used to compare the median difference between groups

may be shared with other developing nations. Previous Egyptian studies confirmed that elevated admission glucose level is a strong predictor of short-term adverse outcomes in patients with AMI but no previous studies in our country regarding the impact of $\mathrm{AH}$ on AAR, FIS, or SI which is the major concept of our research $[10,11]$.

An Indian study had addressed the association of AHG and increased myocardial damage evidenced by cardiac biomarkers and echocardiographic derived data but without using CMR modality. In that study, increased admission plasma glucose was associated with lower LVEF, higher WMSI, and higher CKMB in STEMI patients, all in line with our results except for LVEF which had an insignificant association with AHG in our studied population [12].

One major finding in our study is that AAR was positively correlated with APG; this is explained by the fact that acute hyperglycemia may increase the inflammatory response during STEMI and could thus influence microvascular permeability and edema [13]. To the limit of our knowledge, few studies investigated the impact of stress hyperglycemia on AAR [14]. Another study found no association between AHG and FIS [15].

Moreover, admission plasma glucose associated with larger FIS estimated by CMR and assessed by delayed enhancement (DE) score in acute STEMI patients [16].
However, the mechanism by which admission hyperglycemia adversely affect myocardial FIS is not well understood. But it was proved that admission hyperglycemia strongly predicted the reduction of epicardial flow in the infarct-related vessel before reperfusion therapy in patients with STEMI [17]. Moreover, admission hyperglycemia may be a consequence of large infarction size rather than the cause; as infarction increases in size, more catecholamines are secreted, which in turn affect fatty acids and glucose secretion [18].

Several studies have investigated the clinical impact of LGE on patient outcome after AMI. However, few studies have evaluated clinical factors or laboratory parameters that may be predictive for the development of LGE. Identification of such predictors for the development of LGE post-AMI may facilitate early risk stratification, even before cardiac imaging. In line with our study, a previous one supported that delayed presentation was predictor for larger infarction size [19]. In contradiction to our results, a previous one found no significant relationship between the time to PCI and infarction size [20]. Supporting to our data, a previous study revealed that high CK-MB values were independent predictors of LGE [21]. Similarly, Klug et al. observed a correlation between CK value and infarct size in reperfused STEMI [22].

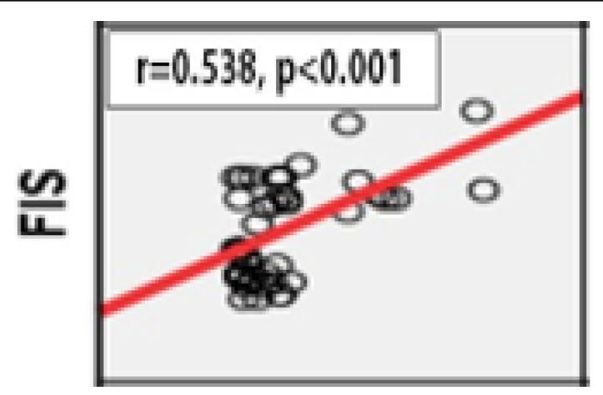

APG

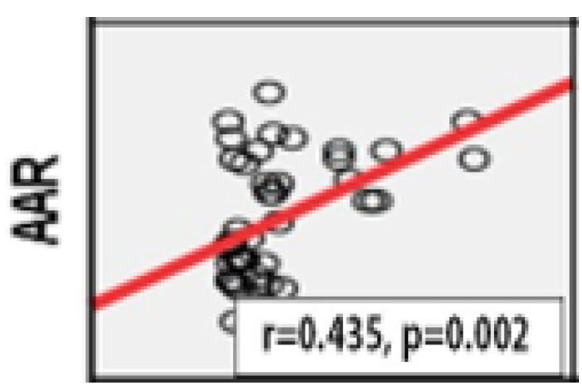

APG

Fig. 2 Correlation between admission blood glucose and final infraction size (FIS) and area at risk (AAR) 
Table 4 The multivariate linear regression analysis of the factors affecting FIS

\begin{tabular}{|c|c|c|c|c|}
\hline & Estimate & SE & $t$ stat & $p$ value \\
\hline Intercept & $5.87(1.05-14.18)$ & 1.14 & 4.87 & $=0.011$ \\
\hline Age/years & $0.02(-0.34-0.37)$ & 0.23 & 0.09 & $=0.928$ \\
\hline Heart rate (beat/min) & $0.13(-0.15-0.41)$ & 0.12 & 0.96 & $=0.343$ \\
\hline Chest pain duration (h) & $1.04(0.28-1.80)$ & 0.41 & 2.75 & $=0.009$ \\
\hline APG (mg/dl) & $0.08(0.04-0.11)$ & 0.02 & 4.09 & $<0.001$ \\
\hline Peak CK-MB (ng/ml) & $0.16(0.08-0.24)$ & 0.04 & 3.99 & $<0.001$ \\
\hline Sum ST elevation & $0.71(0.38-1.04)$ & 0.16 & 4.35 & $<0.001$ \\
\hline Max ST elevation & $2.57(1.03-4.10)$ & 0.76 & 3.38 & $=0.002$ \\
\hline Echo WMSI & $-1.73(-5.29-1.95)$ & 0.91 & 0.84 & $=0.624$ \\
\hline CMR WMSI & $1.89(1.01-3.51)$ & 0.18 & 4.62 & $=0.005$ \\
\hline Infarction site (anterior) & $4.07(-2.84-10.98)$ & 3.40 & 1.29 & $=0.240$ \\
\hline
\end{tabular}

$p$ value $<0.05$ is significant

$A P G$ admission plasma glucose, $C K$ creatinine kinase, $C M R$ cardiac magnetic resonance, Max ST elevation maximum ST elevations, Sum ST elevation summation of ST elevations, WMSI wall motion score index

In this small study, we tried to start highlighting the ability of CMR-derived prognosticators to detect the impact of AHG on FIS and AAR in this cohort of patients in Egypt without neglecting less accurate but more available and less costly ECG and echocardiographic data.

\section{Study limitations}

Our study did not include any data regarding details of insulin therapy given to the patients with admission hyperglycemia which may influence the FIS and AAR. So, it is recommended to investigate the role of glucoselowering therapy in hyperglycemic patients on FIS. Our study interpretation should be applied to low- and moderate-risk STEMI patients as high-risk STEMI (e.g., higher Killip class or cardiogenic shock) patients are excluded because they were not able to continue the procedure. Financial problems and lack of funding support restricted the sample size which decreases the validity of the studied variables.

\section{Conclusions}

Admission plasma glucose positively correlates with CMR estimated FIS and AAR in successfully reperfused STEMI.

\footnotetext{
Abbreviations

AAR: Area at risk; AH: Admission hyperglycemia; APG: Admission plasma glucose; CK: Creatine kinase; CMR: Cardiac magnetic resonance; DE: Delayed enhancement; ECG: Electrocardiogram; EF: Ejection fraction; FIS: Final infarction size; Gd-DTPA: Gadolinium diethylenetriaminepentaacetic acid; HbA1c: Glycated hemoglobin; LBBB: Left bundle branch block; LDL: Low density lipoprotein; LGE: Late gadolinium enhancement; LV: Left ventricular; LVEDV: Left ventricular end-diastolic volume; LVESV: Left ventricular endsystolic volume; Max STE: Maximum ST segment elevation; PPCI: Primary percutaneous coronary intervention; PSIR: Phase sensitive inversion recovery; SD: Standard deviation; SPECT: Single-photon emission computed tomography; STEMI: ST-elevation myocardial infarction; STIR: Short-tau
}

inversion-recovery turbo-spin echo sequence; Sum STE: Summation of ST segment elevation; WMSI: Wall motion score index

\section{Acknowledgements}

We thank the patients and their families for their participation in this study.

\section{Authors' contributions}

1. T.M: the acquisition and analysis of data, approved the submitted version, and agreed both to be personally accountable for the author's own contributions and to ensure that questions related to the accuracy or integrity of any part of the work are appropriately investigated, resolved, and the resolution documented in the literature. 2. M.A: put the concept of the research, approved the submitted version, and agreed both to be personally accountable for the author's own contributions and to ensure that questions related to the accuracy or integrity of any part of the work are appropriately investigated, resolved, and the resolution documented in the literature. 3. R.A: put the design of the research, approved the submitted version, and agreed both to be personally accountable for the author's own contributions and to ensure that questions related to the accuracy or integrity of any part of the work are appropriately investigated, resolved, and the resolution documented in the literature. 4. H.I: revision of the work, approved the submitted version, and agreed both to be personally accountable for the author's own contributions and to ensure that questions related to the accuracy or integrity of any part of the work are appropriately investigated, resolved, and the resolution documented in the literature. 5. D.H: interpretation of data, approved the submitted version, and agreed both to be personally accountable for the author's own contributions and to ensure that questions related to the accuracy or integrity of any part of the work are appropriately investigated, resolved, and the resolution documented in the literature. All authors have read and approved the manuscript.

\section{Funding}

None.

Availability of data and materials

- Patients are recruited from those who were admitted to Critical Care Unit, Internal Medicine Department, Assiut University.

- CMR was performed in Radiology Department, Assiut University.

- Gadolinium used in CMR was on our expense as there was no any funding support.

Ethics approval and consent to participate

"The Committee of Medical Ethics" of Faculty of Medicine, Assiut University, had approved the study with reference no. 17200407.

Written consents were obtained from all patients.

\section{Consent for publication}

Not applicable.

No identifying images nor personal data were included in the manuscript.

\section{Competing interests}

None.

\section{Author details}

${ }^{1}$ Internal Medicine Department, Assiut University Hospitals, Faculty of Medicine, Assiut University, Assiut 71511, Egypt. ${ }^{2}$ Assuit University, Assuit, Egypt.

Received: 20 July 2020 Accepted: 3 August 2020

Published online: 13 October 2020

References

1. Organization WH (2017) Fact sheet: the top 10 causes of death. World Health Organization, Geneva, Switzerland

2. Cinar H, Akkan Avci MG, Avci BS, Comertpay E, Satar S (2019) Does stress hyperglycemia affect mortality? Acute myocardial infarction-case control study Archives of medical sciences Atherosclerotic diseases 4:e201

3. Aziz EF, Javed F, Alviar CL, Herzog E. Triple vessel coronary artery disease presenting as a markedly positive stress electrocardiographic test and a negative SPECT-TL scintigram: a case of balanced ischemia. Heart Int 2011; 6: hi. 2011. e2022 
4. Krittayaphong R, Laksanabunsong P, Maneesai A et al (2008) Comparison of cardiovascular magnetic resonance of late gadolinium enhancement and diastolic wall thickness to predict recovery of left ventricular function after coronary artery bypass surgery. J Cardiovasc Magn Reson 10:41. https://doi. org/10.1186/1532-429X-10-41

5. Koliaki C, Liatis S, Kokkinos A (2019) Obesity and cardiovascular disease: revisiting an old relationship. Metabolism 92:98-107

6. Cockcroft DW, Gault H (1976) Prediction of creatinine clearance from serum creatinine. Nephron 16:31-41

7. Cottin Y, Touzery C, Guy F et al (1999) MR imaging of the heart in patients after myocardial infarction: effect of increasing intersection gap on measurements of left ventricular volume, ejection fraction, and wall thickness. Radiology 213:513-520

8. Alegria JR, Miller TD, Gibbons RJ, Yi Q-L, Yusuf S (2007) Infarct size, ejection fraction, and mortality in diabetic patients with acute myocardial infarction treated with thrombolytic therapy. Am Heart J 154:743-750

9. Shah AM, Uno H, Køber $L$ et al (2010) The inter-relationship of diabetes and left ventricular systolic function on outcome after high-risk myocardial infarction. Eur J Heart Fail 12:1229-1237

10. Zaghla HE, Elbadry MA, Ashour AM, Abdelfatah MM (2014) Influence of admission blood glucose and hemoglobin A1c on outcome of acute myocardial infarction. The Egyptian Journal of Internal Medicine 26:21

11. Khalfallah M, Abdelmageed R, Elgendy E, Hafez YM (2019) Incidence, predictors and outcomes of stress hyperglycemia in patients with ST elevation myocardial infarction undergoing primary percutaneous coronary intervention. Diab Vasc Dis Res 1479164119883983

12. Chakraborty S, Majumder B, Sarkar D, Chatterjee S (2017) A simple noninvasive ECG technique to localize culprit vessel occlusion site in STelevation myocardial infarction (STEMI) patients. J Clin Exp Cardiolog 8:2

13. Marfella R, Siniscalchi M, Esposito K et al (2003) Effects of stress hyperglycemia on acute myocardial infarction: role of inflammatory immune process in functional cardiac outcome. Diabetes Care 26:31293135

14. Lønborg J, Vejlstrup N, Kelbæk H et al (2014) Impact of acute hyperglycemia on myocardial infarct size, area at risk, and salvage in patients with STEMI and the association with exenatide treatment: results from a randomized study. Diabetes 63:2474-2485

15. Ota S, Tanimoto T, Orii M et al (2015) Association between hyperglycemia at admission and microvascular obstruction in patients with ST-segment elevation myocardial infarction. J Cardiol 65:272-277

16. Cochet A, Zeller M, Lalande A et al (2008) Utility of cardiac magnetic resonance to assess association between admission hyperglycemia and myocardial damage in patients with reperfused ST-segment elevation myocardial infarction. J Cardiovasc Magn Reson 10:2

17. Timmer JR, Ottervanger JP, de Boer M-J et al (2005) Hyperglycemia is an important predictor of impaired coronary flow before reperfusion therapy in ST-segment elevation myocardial infarction. J Am Coll Cardiol 45:999-1002

18. Capes SE, Hunt D, Malmberg K, Gerstein HC (2000) Stress hyperglycaemia and increased risk of death after myocardial infarction in patients with and without diabetes: a systematic overview. Lancet 355:773-778

19. Nallamothu BK, Bradley EH, Krumholz HM (2007) Time to treatment in primary percutaneous coronary intervention. N Engl J Med 357:1631-1638

20. Schömig A, Ndrepepa G, Mehilli J et al (2003) Therapy-dependent influence of time-to-treatment interval on myocardial salvage in patients with acute myocardial infarction treated with coronary artery stenting or thrombolysis. Circulation 108:1084-1088

21. Abanador-Kamper N, Kamper L, Vorpahl M et al (2017) Clinical predictors for the manifestation of late gadolinium enhancement after acute myocardial infarction. Medicine 96

22. Klug G, Mayr A, Mair J et al (2011) Role of biomarkers in assessment of early infarct size after successful p-PCI for STEMI. Clin Res Cardiol 100:501-510

\section{Publisher's Note}

Springer Nature remains neutral with regard to jurisdictional claims in published maps and institutional affiliations.

\section{Submit your manuscript to a SpringerOpen ${ }^{\circ}$ journal and benefit from:}

- Convenient online submission

- Rigorous peer review

- Open access: articles freely available online

High visibility within the field

- Retaining the copyright to your article

Submit your next manuscript at $\boldsymbol{\nabla}$ springeropen.com 